\title{
Mathematical Modelling for the Social Impact to Energy Efficiency Savings ${ }^{\text {th }}$
}

\author{
Uduakobong E. Ekpenyong $^{1 *}$, Jiangfeng Zhang ${ }^{2}$, Xiaohua Xia ${ }^{1}$ \\ ${ }^{1}$ Centre of New Energy Systems, Department of Electrical, Electronic and Computer \\ Engineering, University of Pretoria, South Africa \\ ${ }^{2}$ Department of Electronic and Electrical Engineering, University of Strathclyde, United \\ Kingdom
}

\begin{abstract}
In this paper, a mathematical model is formulated to quantify the social impact an individual has on his/her community when he/she performs any energy efficiency project and transmits that information to his/her neighbours. This model is called the expected power savings model; it combines direct and indirect expected power savings of the energy efficiency project for each individual within the network. The indirect savings are quantified through the social interactions people in the network. The example used in this paper illustrates the effectiveness of the model by identifying the households who should receive free solar water heaters based on their influence through interactions in their community. Two case studies are considered in this paper, single and multiple sources case studies. In the multiple source case study, the results show that it is not necessarily the people with the highest connections who provide the maximum expected power savings.
\end{abstract}

Keywords: Energy efficiency, Social impact, Social network

\section{Introduction}

In all energy efficiency projects, humans are the common factor and they play a major role towards the success of any project. It then makes sense that, humans are the ones to target when campaigning for change of any

\footnotetext{
${ }^{2}$ A preliminary version of this article has been presented in the International Energy Program Evaluation Conference, Rome, Italy, 12 - 14 June, 2012.

*Corresponding author: Tel.: +27 12 4204341; Fax: +27 123625000
} 
kind concerning energy efficiency. The authors of [1] suggest that interactions among residents in a network increase energy savings which maybe more cost effective than physical renovations. There are several studies that show the impact social networks play in reducing energy consumption [2][5]. Identifying the people who will implement the energy efficiency projects and afterwards spread the news encouraging free riding on energy efficiency programs.

Energy savings can be used to identify people who will propagate their savings information. Energy savings consist of two parts: direct and indirect savings. The direct savings refer to the savings that are measurable or observable and can be determined by various measurement and verification techniques [6]. The indirect savings refer to savings additional to direct savings, which are achieved by social interactions of people in a network. This social interaction is classified under the performance efficiency of the POET classification [7]. The indirect savings can help identify people with most influence in their network through their transfer of information about their energy efficiency projects. It can also help calculate the expected power saved from an energy efficiency project, predict the optimal location for an energy efficiency project that will yield maximum expected savings and calculate the acceptability of a project within a network.

The mathematical model proposed in this article (known from here on as the expected power saving model) is loosely related to the pinning control of complex networks. Pinning control is when a network cannot synchronise on its own and some controllers are applied to selected nodes in the network to force the network to synchronise [8]-[10]. Physically this means the model identifies people who should implement energy efficiency projects initially in their network and these people encourage everyone to implement those projects. This helps saves money and encourages free riding. Free riders are defined as those people in a energy efficiency program who would have installed the same energy efficiency measures even if there had been no program [11], [12].

The indirect savings is defined as that contribution a person makes to encourage other people to implement energy efficiency projects. This contribution is represented as a function of the direct savings. The indirect savings helps determine the people with the most influence through information transfer and selecting them for energy efficiency projects. After they implement the projects, they are able to spread the information about the project to others in so doing it will encourage people to implement efficiency projects.

Identifying people to receive the project can be seen as a good way for the 
neighbours to free ride on the information they have received from the person chosen to receive the energy efficiency project. Rather than the neighbours going through the process of finding ways of reducing their electricity use which could be time consuming and costly, receiving the information from their neighbours gives them confidence about the project and hence encourage them to implement that project. An example of this type of search through free riding is used in [13]

The indirect savings calculated in this article make use of the knowledge of small world phenomenon of social networks [14]-[18] and information entropy [19]. Milgram's experiment shows that any two people in any part of the world are connected by an average of six intermediate people [14], [15]. The latest version of the small world experiment on Facebook (an online social network website) reports that the average number of acquaintances separating two people is 4.74 [20].

A case study on a community of fifty-six households is performed to identify the household that have more influence through social interactions in their network. The discovery of the household that will spread the most information is done using the expected power saving model proposed in this paper. Results from the case study determine the suitable households to receive the solar water heaters. Intuitively, when faced with the choice of giving solar water heaters to more than one household, the households that have the highest number of people connected to them is chosen but this paper has proven this assumption false through the expected power saving model.

The layout of the paper is as follows. Section 2 gives a quick background on social networks and information transfer. In Section 3 a mathematical model is formulated to calculate the expected power savings of an energy efficiency project for single and multiple sources of information transfer within network. An example to test the model is given in Section 4 and the results of the findings are discussed in Section 5. Section 6 gives the conclusion and areas of further research.

\section{Background}

\subsection{Social network}

The authors of [21] and [22] point out that the existence of connection among people does not mean that they are aware of them. Hence the exclamation of "it's a small world!" when two people meet who previously do not know each other but have a mutual acquaintance. [3] shows that social networks promote energy efficiency savings. This paper explores the existence 
of the connections in a network, then quantifies the expected power savings through interactions.

Mathematical formulation of a social network problem in [16] and [17] gives the calculation of connectivity of people within a network. For simplicity, the network in this paper is assumed to be represented as a connected graph consisting of nodes with unweighted and undirected edges. The node degree $k_{l}$ of the node $l$ is the number of edges linking with node $l$. The node degree $k_{l}$ shows the interaction and information sharing among the nodes in the network. The network degree distribution $D$ is defined as

$$
D=\frac{1}{N} \sum_{l=1}^{N} k_{l}=\frac{2 E}{N},
$$

where $N$ is the total number of nodes in the network and $E$ is the total number of edges in the network. The degree distribution $D$ of any network gives the average node degree of all the nodes in the network.

The degree of connection is derived from the degree of separation that is explained by Milgram's experiment, it shows that anyone can be connected to any other person on the planet through a chain of acquaintances which has an average of five intermediaries [14]. This means that when $i$ has one degree of connection to $j$ then $d_{i, j}=1$, and they are directly connected to each other. Similarly, two people are said to have two degrees of connection $d_{i, j}=2$ if they are connected by one intermediary. The set $M_{l}$ is defined as the set of people directly connected to $l$. The average degree of connection for the entire network is the characteristic path length of that network. Characteristic path length $L$ is defined as the average number of edges that must be traversed in the shortest path between any two pairs of nodes in the graph, it is a measure of the global structure of the graph. The characteristic path length is defined in [17] as

$$
L=\frac{1}{N(N-1)} \sum_{i=1}^{N} \sum_{j=1}^{N} d_{i, j} .
$$

The degree distribution helps determine the functional probability of any individual in the network, this will be explained in the next subsection while the characteristic path length helps determine the conditional probability which will also be explained in the next subsection.

\subsection{Information transfer}

The diffusion of information depends on the new idea and the members of the social network [21]-[23]. In literature [24]-[26], it is assumed that the 
information passed from person A to person B is the full information and B understands the information. In real life this is not the case, there is always some information that is lost during transmission. The application of the entropy of information has been successfully used in different fields of complex networks such as water supply [27]-[28], ecology [29], [30], evaluation of alternative measures of new energy saving technologies [31] and it shows the possibility of defining and quantifying information transfer among people.

The functional probability $p(i)$ is proportional to the ratio of the node degree $k_{i}$ of $i$ to the total number of edges $E$ in the network. This gives the probability that a node is in the network and connected to other nodes. The conditional probability $p_{i}(j)$ refers to the probability that node $i$ is connected to node $j$ through at most four intermediaries. The joint probability $p(i, j)$ is the probability that the information regarding an energy efficiency project has been transferred from the end user $i$ who performs the project to his neighbour $j$.

The relationship between $p(i), p_{i}(j)$ and $p(i, j)$ in [19] is given as:

$$
\begin{gathered}
p(i, j)=p(j, i)=p(i) p_{i}(j), \\
\sum_{i=1}^{N} p(i)=\sum_{1 \leq j \leq N, j \neq i} p_{i}(j)=\sum_{1 \leq i \leq N, i \neq j} \sum_{1 \leq i, j \leq N, j \neq i} p(i, j)=1 .
\end{gathered}
$$

The entropy in information theory is defined as a measure of information, choice or uncertainty. The entropy gives the quantity of information transferred within the network. The entropy $H(i)$ for a single source of information is calculated as [19]

$$
H(i)=-\sum_{1 \leq j \leq N, j \neq i} p(i, j) \log _{2} p_{i}(j)
$$

\section{Methodology and mathematical model}

Energy efficiency projects are implemented by humans, therefore quantifying the social impact through social interactions will give the total expected power saving in every energy efficiency project. Social influence of an individual is dependent on the peer-to-peer interactions; this can highlight the most influential people in a network and thus reveal to energy planners the people to target in the propagating information about energy efficiency projects. Identifying people who will spread the information about the energy efficiency project to the network fastest is important because this 
will help change people's behaviour towards energy conservation and thus increase energy savings at little or no cost.

The mathematical model of the expected power savings calculates the combined direct and indirect savings of the energy efficiency project. In the model, the physical distance between two people is not considered, two people are said to be connected if there is a mutual acknowledgement of friendship between them. The nodes represent the households and the edges represent the connection between two households. The mathematical model of the expected power savings considers two scenarios; when there is focus on one or multiple end users to transfer information to the rest of the network. This model will try to dispute the intuitive believe that people with the highest node degree will spread the most information in the network.

Assume that the $i$-th end user is the only one in the network who performs an energy efficiency project, the expected power saving is calculated as:

$$
E P S=S_{i}+\sum_{1 \leq j \leq N, j \neq i} S_{j, i}^{\text {indirect }},
$$

where $S_{i}$ is the direct savings from the energy efficiency project that the $i$-th end user implements. The calculations of direct savings are not a major contribution of this research hence in the case study, the direct savings are given. $S_{j, i}^{\text {indirect }}$ is the indirect saving of the $j$-th end user that is affected by the social impact of the $i$-th source node. The source node is a representation of an end user that performs an energy efficiency project and is able to transfer information about the project to other nodes.

Now consider the case where the network has more than one end user implementing energy efficiency projects. The expected power saving for multiple sources is calculated as:

$$
E P S=\sum_{i \in I} S_{i}+\sum_{i \in I} \sum_{1 \leq j \leq N, j \notin I} S_{j, i}^{\text {indirect }}
$$

where $I$ is the set of source nodes in the network. Note that the summation $\sum_{1 \leq j \leq N, j \notin I} S_{j, i}^{\text {indirect }}$ excludes the case that one source is influenced by another source. This is because a source node already has its direct saving therefore, any information that is transferred from another source will have no effect and hence no indirect savings

The entropy of information theory is applied to information sharing of energy efficiency projects within a network. The higher the entropy the more the information about the energy efficiency projects is expected to be transferred within the network. With this knowledge, the expected indirect 
savings for a single source case is defined as

$$
\sum_{1 \leq j \leq N, j \notin I} S_{j, i}^{\text {indirect }}:=H(i) S_{i},
$$

where $H(i)$ is defined as the entropy in equation (5). In the case where more than one end user performs energy efficiency projects, the indirect saving for the multiple sources case follows easily from (8)

$$
\sum_{i \in I} \sum_{1 \leq j \leq N, j \notin I} S_{j, i}^{\text {indirect }}:=\sum_{i \in I} H(i) S_{i} .
$$

Formulae (8) and (9) are applied in (6) and (7) for the single and multiple sources respectively.

In the multiple source case, $H(i)$ in $(9)$ is calculated similarly as the single source case. That is,

$$
H(i)=-\sum_{1 \leq j \leq N, \quad j \notin I} p(i, j) \log _{2} p_{i}(j)
$$

From (5) the above (10) can also be written as

$$
H(i)=-\sum_{1 \leq j \leq N, \quad j \notin I} p(i) p_{i}(j) \log _{2} p_{i}(j) .
$$

It turns out that the single source case in equation (6) is a special case of equation (7) for multiple sources. Therefore, we will not distinguish the single source and multiple source cases in the following calculations of $p(i)$ and $p_{i}(j)$.

The functional probability $p(l)$ for any node $l$ gives the likelihood that the node has a node degree $k_{l}$ out of the network degree distribution in an $N$ total number of nodes in the network. The functional probability $l$ is defined as

$$
p(l)=\frac{k_{l}}{D N}, \text { for } l=1,2, \ldots, N .
$$

Now consider the calculation of $p_{i}(j)$ where $i \in I$ and $j \notin I$, note that $p_{i}(j)$ is the quantitative value for the connectivity of nodes within the network.

The further people are from one another the impact of information transferred is reduced, as shown in Figure 1 where the boxes represent the information transferred from the source. As the boxes move further from the source the lighter they are meaning their impact on the receiving node is 


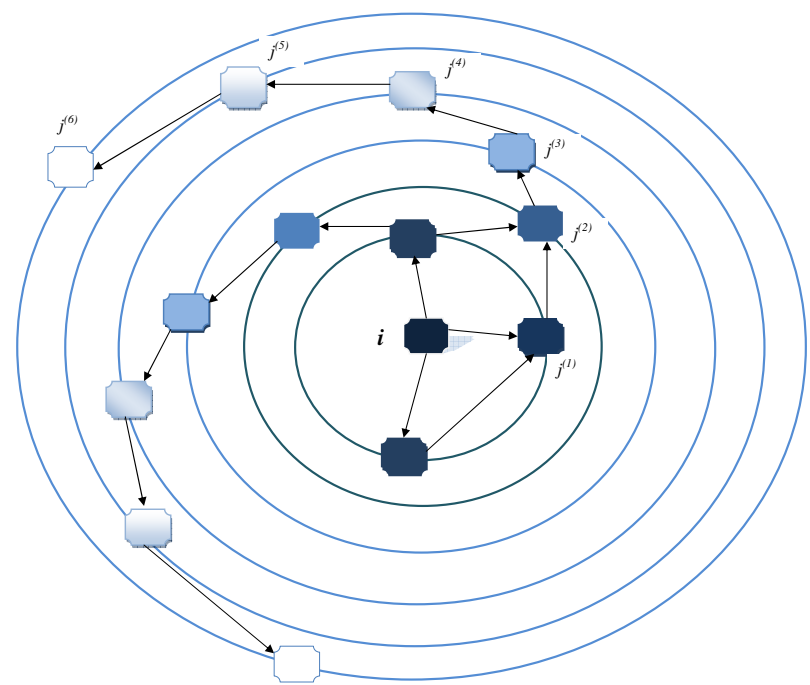

Figure 1: Information transfer

reduced. The greater the intermediaries between the source node and the receiver of the information, the smaller the information is transferred. In the calculation of $p_{i}(j)$ for a medium sized network, we consider only the case that $j$ is connected to the source node $i$ with degree of connection of at most four. This is a good approximation to the latest research on social networks that an individual is separated from any one in the world by an average characteristic path length $L=4.74$ people [20].

In a network, the conditional probability $p_{i}(j)$ that an information source node $i$ can transfer information to another individual $j$ depends on how these two nodes are connected to other nodes. Note that information transferred along shorter paths are always dominant when compared to the information transferred along longer paths. Therefore, it is reasonable to consider only information transferred along shortest paths when considering the definition of $p_{i}(j)$. This is to say, information transferred along longer paths will be ignored, and if the shortest path between $i$ and $j$ is not unique, then information transferred along all the shortest path will be added together. Physically, this translates to the fact that the more a person hears about the advantages of a product from more than one friend, the more likely he will be convinced to acquire that product. Therefore the conditional probability does not only focus on the source nodes who transmits the information but also on the sink node's different access to the information. The following cases are considered in the definition of $p_{i}(j)$. 
Case I: Assume that the degree of connection of node $j$ with node $i$ is one, that is node $j$ is directly connected with node $i, d_{i, j}=1$. Then $p_{i}(j)$ is defined as

$$
p_{i}(j):=\frac{1}{k_{i} k_{j}} .
$$

Case II: Assume that the degree of connection of node $j$ with node $i$ is two, that is $j \notin M_{i}$. Now we define the $p_{i}(j)$ as

$$
p_{i}(j):=\frac{1}{k_{i} k_{j}} \sum_{q \in M_{i} \cap M_{j}} \frac{1}{k_{q}} .
$$

In (14) the second degree of information transfer is dependent on the information already transferred from the source node to the first degree node $q \in M_{i} \cap M_{j}$. The second degree node $j$ treats the first degree node $q$ as its source of information and the information obtained is dependent on the amount of information that is passed to $q$ from the source node $i$. This means that $q$ transfers the information he/she obtained from $i$ to $j$. This shows the continuity of information transfer among nodes in the network. The sum of the probability of total number of nodes $q$ between $i$ and $j$ indicates that when one hears about a lifestyle change from several friends the higher the chances of that person adopting that lifestyle change. This can also been seen from the receivers point of view for example, the more people tell him/her about their savings through buying some retrofitting of their home, the more likely this person will change in order to obtain those savings. If $j$ decides to adopt to this lifestyle in order to save, it does not mean that $j$ will automatically buy all the retrofits that all his/her friends tell him/her as this will not be realistic or cost effective. This means that the more information $j$ obtains about savings from his/her friend the more likely he/she will be willing to change to that lifestyle. In addition, it confirms that the social impact $i$ has on $j$ through information transfer is lesser than the impact $i$ has on $q$ and this depicts real life scenarios where the influence of one's friends are greater than the influence of a friend of a friend [3] . Case III and IV follow the same thought pattern as case II.

Case III: Assume that the degree of connection of node $j$ with node $i$ is three, that is $d_{i, j}=3$, then $p_{i}(j)$ is defined as

$$
p_{i}(j):=\frac{1}{k_{i} k_{j}} \sum_{(q, r)} \frac{1}{k_{q} k_{r}}, \quad q \in M_{i}, r \in M_{j}, q \in M_{r} .
$$

Case IV: Assume that the degree of connection of node $j$ with node $i$ is 
four that is $d_{i, j}=4$, then $p_{i}(j)$ is defined as

$$
p_{i}(j):=\frac{1}{k_{i} k_{j}} \sum_{(q, r, s)} \frac{1}{k_{q} k_{r} k_{s}}, \quad q \in M_{i}, r \in M_{q} \cap M_{s}, s \in M_{j} .
$$

Case $V$ : Assume that the degree of connection of node $j$ with node $i$ is greater than four, the conditional probability is assumed to be negligible and therefore

$$
p_{i}(j):=0 .
$$

The practicality of (13) - (17) is that when a person performs an energy efficiency project, the information he/she transfers to the network is dependent on how many neighbour he/she has and how many neighbours his/her neighbours have too. The conditional probabilities measure the quantity of information transferred from the end user $i$ to his/her neighbour $j$. As information is never fully transferred, the more people between $i$ and $j$ the less the quantity of information will be transferred. The condition probability ensures that all the people who could possibly receive information from the end user do. And it aids in the calculation of the information entropy that determines the influence a person has on the rest of the network. The expected energy savings model presented here incorporates the quantity of information transferred within the network that evaluates the indirect savings of an individual and in turn determine the expected energy savings.

\section{Case study}

The South African government has partnered with the local utility company Eskom to provide some limited free low pressure solar water heaters to households within South Africa. When the households to receive the free solar water heaters are chosen, a member of the household has to be present while the installation is carried out. After the installation, a brief description of the solar water heater and lessons on how to use the heaters are given. The benefits of the solar water heater is highlighted to the member of the household [32]. This is done with the expectation that the person talks about the efficiency of the heater to his/her friends. The transfer of such information leads to more people purchasing the solar water heaters.

People are connected to each other through various means and as such information is transferred from one household to another. The reasons any two households are connected to each other are based on different factors such as environmental proximity, members of the same organisation, have 
children in the same school or work at the same office. In this research, a survey is carried out on a group consisting of fifty-six households from the same church organisation to obtain data for the social network graph. Each household is given a questionnaire to write out the names of other households they consider as friends within the group. After the necessary information has been collected, an adjacency matrix is constructed. The criterion for the graph is that two households must acknowledge that they are friends with each other before an edge can be drawn between them.

In order to calculate the expected power savings, the following assumptions are made,

1. Each household is assumed to use their electric heaters at about the same time through out the community, for example at both the morning and evening peak hours, which are between 07:00 - 10:00 and 18:00 - 20:00 hours respectively. These peak hours are adopted from the HomeFlex Eskom time of use tariff [33].

2. Installation of each solar water heater will save at least $2 \mathrm{~kW}$ of power when comparing with turning on an electric water heater [33]. This means that the direct savings for this paper $S_{i}=2$.

3. The distance and type of relationship between each household is not considered in this problem and hence the network problem is considered to be unweighted and undirected.

4. For the duration of the study there are no new members introduced into the network and none of the members leave the network.

There are two case studies presented in this paper, the first case is when there is only one person to be given a new solar water heater and the second case is when there is more than one person to give the solar water heater. The installation of the solar heaters is to promote renewable technology and to encourage people to buy the solar water heaters. The use of the solar water heaters reduces the electricity bills and consumption electricity of the entire community. In order to maximise the indirect savings due to social impact, the criteria for houses to receive free solar water heaters will be based on how much power is saved and how much impact these households have on their community. As the direct savings is fixed, the indirect savings will determine the person who has the most expected power savings.

\section{Results and discussions}

In general, a social network graph can be referred to an expression of patterns in relationships among people within a network. The relationships can be based on geographical location, political, kinship, behavioural 


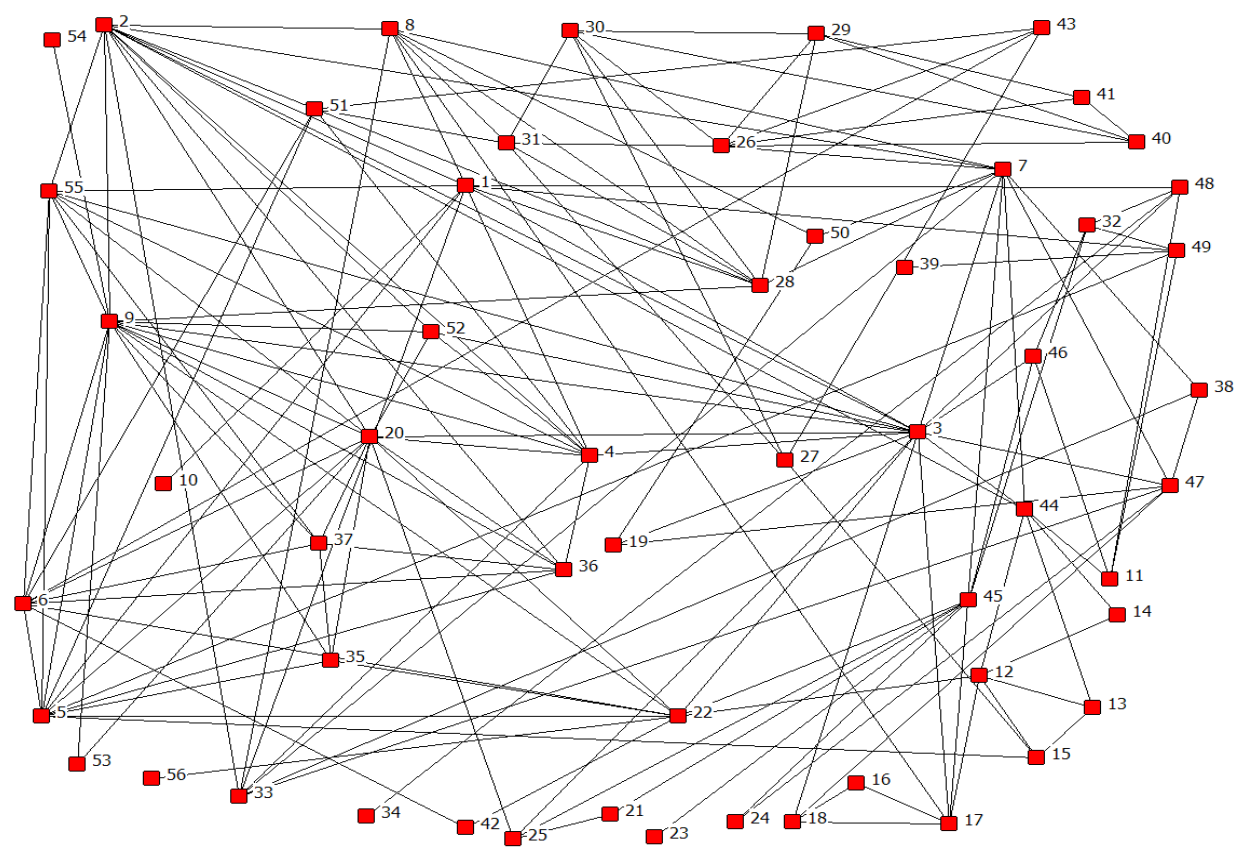

Figure 2: Network graph of the community

interactions, friendship, affiliation or economic to name a few. These relationships among members of a network is used to establish a social network graph. In this paper the relationship is based on mutual acknowledgement of friendships among households in the network. The network graph is constructed from nodes (households) and edges (relationships). Household $i$ and $j$ must agree that they know each other and are friends before a link (edge) is made between them. How each friendship is formed and the level of friendship such as close, very close and acquaintances are not covered in the scope of this paper. The network graph of Figure 2 based on the adjacency matrix of the 56 members of the community. The graph is an unweighted and undirected graph, that is when two households are connected $d_{i, j}=d_{j, i}=1$.

The average number of nodes that any node is connected, which is the network degree $D$ of (1), is 5.66. This means that a person is connected to one-tenth of the total population of the network on average. This shows that people are heavily connected to one another in this network. The average degree of connection of the network $L=2.75$, this corresponds with the definition of the assumptions of the conditional probability formula (17) 


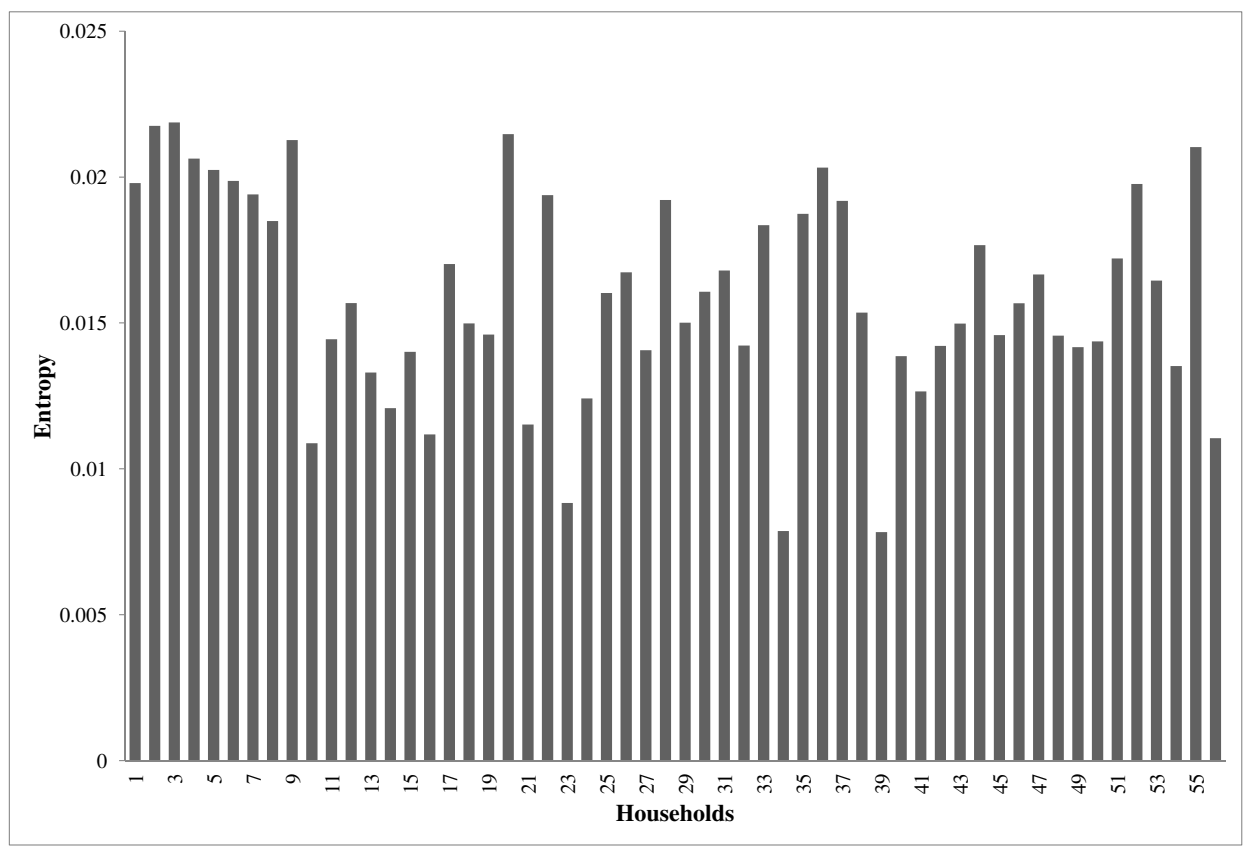

Figure 3: Entropy of all nodes

and the latest findings that any two people chosen at random will have at most 4 intermediaries between them. The network used in the case study shows that it is a real life a small work network in with a small mean path length and large network degree.

\subsection{Case study I}

Assume that there is only one solar water heater to be given out for free. In order to identify the best household that will qualify for the free heater, the expected power savings for single source nodes of all the 56 people are calculated using the entropy, the indirect savings and total expected power savings using equations (5), (8) and (6) respectively. The person with the biggest total expected power savings is therefore the person who will transmit the information about the solar water heater most effectively and through his/her broadcasting about the advantages solar heaters will encourage other people to buy their own heaters. The results of the household expected power savings and information entropy from the highest to the lowest are shown in Table 1 and Figure 3 respectively.

The results show that node 3 has the highest entropy, this means that it has the highest possibility of transmitting the information about the solar 
Table 1: Node degrees and expected power savings for single source of information

\begin{tabular}{l|llllllll}
\hline Household/node & 3 & 2 & 20 & 9 & 55 & 4 & 36 & 15 \\
Node degree $\left(k_{i}\right)$ & 17 & 13 & 14 & 15 & 9 & 10 & 7 & 11 \\
EPS $(\mathrm{kW})$ & 2.055 & 2.054 & 2.054 & 2.053 & 2.053 & 2.052 & 2.051 & 2.051 \\
\hline Household/node & 6 & 1 & 52 & 7 & 22 & 28 & 37 & 35 \\
Node degree $\left(k_{i}\right)$ & 10 & 11 & 5 & 10 & 8 & 6 & 5 & \\
EPS $(\mathrm{kW})$ & 2.05 & 2.049 & 2.049 & 2.049 & 2.048 & 2.048 & 2.048 & 2.047 \\
\hline Household/node & 8 & 33 & 44 & 51 & 17 & 31 & 26 & 47 \\
Node degree $\left(k_{i}\right)$ & 8 & 7 & 7 & 5 & 6 & 6 & 7 & 7 \\
EPS $(\mathrm{kW})$ & 2.046 & 2.044 & 2.043 & 2.043 & 2.043 & 2.042 & 2.042 & 2.042 \\
\hline Household/node & 53 & 30 & 25 & 12 & 46 & 38 & 29 & 18 \\
Node degree $\left(k_{i}\right)$ & 2 & 6 & 4 & 6 & 4 & 3 & 5 & 4 \\
EPS (kW) & 2.041 & 2.040 & 2.040 & 2.039 & 2.039 & 2.038 & 2.038 & 2.037 \\
\hline Household/node & 43 & 19 & 45 & 48 & 11 & 50 & 32 & 42 \\
Node degree $\left(k_{i}\right)$ & 4 & 3 & 7 & 5 & 4 & 3 & 4 & 2 \\
EPS (kW) & 2.037 & 2.036 & 2.036 & 2.036 & 2.036 & 2.036 & 2.036 & 2.036 \\
\hline Household/node & 49 & 27 & 15 & 40 & 54 & 13 & 41 & 24 \\
Node degree $\left(k_{i}\right)$ & 5 & 4 & 4 & 4 & 1 & 3 & 3 & 2 \\
EPS $(\mathrm{kW})$ & 2.035 & 2.035 & 2.035 & 2.035 & 2.034 & 2.033 & 2.032 & 2.031 \\
\hline Household/node & 14 & 21 & 16 & 56 & 10 & 23 & 34 & 39 \\
Node degree $\left(k_{i}\right)$ & 2 & 2 & 2 & 1 & 1 & 1 & 1 & 1 \\
EPS (kW) & 2.030 & 2.029 & 2.028 & 2.028 & 2.027 & 2.022 & 2.022 & 2.020 \\
\hline
\end{tabular}

water heater to the rest of the network. Node 3 has 17 connections and the highest entropy $H=0.022$ compared to node 9 with 15 connection and the entropy $H=0.02$. This means that node 3 has the highest influence within the network and thus has the highest social impact on the community. From Table 1, household 2 who has 13 people connected to him has higher expected power savings than household 20 and 9 who have 14 and 15 connections respectively. Since the power saving for every solar water heater is the same, the household with the highest entropy is also the household with the highest expected power saving value. From the results we can see that individual having a high number people connected him does not automatically ensure he/she have the most influence in his/her community through social interactions that will prompt people to save energy. By using the expected power savings model the energy planner has knowledge to some degree the people who are more likely to spread information in a network and thus aiding him in establishing how to encourage those significant 
people to save energy, which in turn will encourage the rest of the network to save energy.

\subsection{Case study II}

If there are more than one household with the highest number of connections it is difficult to determine which household has the most influence on their network. This is where the EPS model gives the best possible solution for the household with the most influence in his/her community in terms of information transfer. Then the expected power saving from each subnetwork consisting of 3 households must be calculated so that the maximum expected power saving can be identified. The expected saving from a 3 -household subnetwork is calculated by using formula (10).

A search of all the possible 3-household subnetwork combinations is done using the brute force search algorithm. The brute force search algorithm exhaustively search through all the possible combinations until the optimal solution is found. In this case the optimal solution is the 3-household subnetwork that has the highest expected power network. The total number 3 -household subnetworks search equals $\left({ }_{56}^{3}\right)=27720$ different combinations. Table 2 lists the expected power savings of the best 10 and worst 10 subnetworks. The combination of households 4, 8, 50 gives the highest expected power savings, which is $6.4 \mathrm{~kW}$ and $38.4 \mathrm{kWh}$ energy saved during the peak periods on an average day. This savings are $6.7 \%$ higher than the worst household combinations. It is worth noting that household 3, 9, 20 are the top 3 households which have the highest connections as individual nodes. However, their combination as a 3 -household subnetwork has only $6.3 \mathrm{~kW}$ expected power saving. Households 39, 55, 56 with the lowest connections have expected power savings of $6.2 \mathrm{~kW}$ which is higher than household 33 , 48,53 with the worst expected power savings. Therefore, in the multiple source case, if households have high connections it does not imply that the expected power savings of those households as a subnetwork will be high too.

The multiple source case study also concludes what the single case study revealed that people will higher connections does not automatically mean they will influence their neighbours more than people with lower connections, hence the need for the EPS model.

\section{Conclusions and future research}

Expected power saving of any energy efficiency project is divided into two parts, which are direct savings calculated from measurement and veri- 
Table 2: Expected power savings (EPS) and expected energy savings (EES) of the best and worst combinations

\begin{tabular}{l|lll|lll}
\hline & Best Combinations & \multicolumn{3}{l}{ Worst Combinations } & \\
\hline $\mathrm{S} / \mathrm{n}$ & Combination & $\begin{array}{l}\text { EPS } \\
(\mathrm{kW})\end{array}$ & $\begin{array}{l}\text { EES } \\
(\mathrm{kWh})\end{array}$ & Combination & $\begin{array}{l}\text { EPS } \\
(\mathrm{kW})\end{array}$ & EES $(\mathrm{kWh})$ \\
\hline 1 & $4,8,50$ & 6.400 & 38.40 & $6,23,52$ & 6 & 36 \\
2 & $4,8,51$ & 6.398 & 38.391 & $9,38,42$ & 6 & 36 \\
3 & $5,10,40$ & 6.398 & 38.386 & $11,27,34$ & 6 & 36 \\
4 & $4,10,33$ & 6.395 & 38.372 & $13,22,32$ & 6 & 36 \\
5 & $4,9,17$ & 6.395 & 38.372 & $17,21,38$ & 6 & 36 \\
6 & $4,10,32$ & 6.395 & 38.371 & $19,24,55$ & 6 & 36 \\
7 & $2,25,38$ & 6.394 & 38.365 & $21,31,53$ & 6 & 36 \\
8 & $5,16,37$ & 6.394 & 38.364 & $26,39,50$ & 6 & 36 \\
9 & $4,8,20$ & 6.394 & 38.363 & $29,46,54$ & 6 & 36 \\
10 & $2,19,36$ & 6.394 & 38.361 & $33,48,53$ & 6 & 36 \\
\hline
\end{tabular}

fication methods and the indirect savings obtained from social interactions. The indirect savings are the additional savings that have not been quantified in literature, however they are added in the model as a function of the direct savings. A mathematical model is formulated to calculate the combined expected direct and indirect savings of energy efficiency projects. The indirect savings quantifies the savings obtained through information transfer among people in the network. Quantifying the social impact each individual has on his/her network enables one to calculate the expected indirect power saved by that individual. This impact can help identify the people to encourage about energy efficiency projects who will in turn encourage the rest of the network. This will save money by reducing people who would join in a mass roll out project helping the utility reduce cost of implementing the project. The model is tested using data obtained from a 56-member community. The results show that for the single source indirect saving calculations the more connections end users have the higher their chances of influence on their neighbours. The multiple source indirect savings are dependent on how many connections a subnetwork of households has and not just the number of connections of each individual household. Therefore, choosing households with the highest connections does not guarantee that the expected power savings will be the optimal result. This means the impact of those households to encourage his/her neighbours may not be as strong as expected if one does not determine the expected power savings first. Further studies on the social impacts of energy efficiency projects will focus on how the different 
levels of relationships and the use of media to propagate energy efficiency projects influences savings in the social network. As this is the first step of the research to identify the people who are have the highest social influence in their communities through the spread the information, it will be good to know how those people can be encouraged to save energy through external sources such as television adverts and government incentives. Different search algorithms will be applied to the social impact problem to reduce the optimisation time since brute force algorithm suffers from combinatorial explosion in large networks.

\section{References}

[1] X. Xu, J.E. Taylor, A.L. Pisello, P.J. Culligan, The impact of placebased affiliation network on energy conservation: an holistic model that integrates the influence of buildings, residents and the neighborhood context, Energy and Buildings 55 (2012) 637-646.

[2] G. Peschiera, J.E. Taylor, The impact of peer network position on electricity consumption in building occupant networks utilizing energy feedback systems, Energy and Buildings 49 (2012) 584-590.

[3] R.K. Jaina, R. Gulbinasb, J.E. Taylor, P.J. Culligan, Can social influence drive energy savings? Detecting the impact of social influence on the energy consumption behavior of networked users exposed to normative eco-feedback, Energy and Buildings 66 (2013) 119-127.

[4] J. Chen, J.E. Taylor, H. Wei, Modeling building occupant network energy consumption decision making: the interplay between network structure and conservation, Energy and Buildings 47 (2012) 515-524.

[5] S. Wassaerman, K. Faust, Social network analysis in the social and behavioural sciences, Social Network Analysis: Methods and Applications, Cambridge University Press, Cambridge, 1994.

[6] International Performance Measurement and Verification Protocol, EVO 10000-1. Energy Valuation Organisation 2007.

[7] X. Xia, J. Zhang, Modelling and control of heavy-haul trains, IEEE Control Systems Magazine 31 (2011) 18-24.

[8] C. Li, G. Chen, Synchronization in general complex dynamical network with coupling delays, Physica A 343 (2004) 263-278. 
[9] F. Sorrentino, M. Bernardo, F. Garofalo, G. Chen, Controllability of complex network via pinning, Physical Review E 75 (2007) 046103.

[10] W. Yu, G. Chen, On pinning synchronization of complex dynamic networks, Automatica 45 (2009) 429-435.

[11] R. Weinstein, R. Scott, C. Jones, Measurement of "free-riders" in energy conservation programs, Evaluation and Program Planning 12 (1989) 121-130.

[12] M. Croucher, Potential problems and limitations of energy conservation and energy efficiency, Energy Policy 39 (2011) 5795-5799.

[13] S.P. King, Search with free riders, Journal of Economic, Behaviour and Organization 26 (1995) 253-271.

[14] J. Travese, S. Milgram, An experimental study of the small world problem, Sociometry 32 (1969) 425-443.

[15] S. Milgram, The small world problem, Psychology Today 1 (1967) 6167.

[16] D.J. Watts, S.H. Strogatz, Collective dynamics of 'small - world' network. Nature 393 (1998) 440-442.

[17] D.J. Watts, Network, dynamics and small world phenomenon, American Journal of Sociology 105 (1999) 493-527.

[18] M.E.J. Newman, D.J. Watts, S.H. Strogatz, Random graphs model for social networks, Proceedings of the National Academy of Sciences 99 (2002) 2566-2572.

[19] C.E.Shannon, A mathematical theory of communication. The Bell Technical Journal 27 (1948) 376-423, 623-656.

[20] L. Backstorm, P. Boldi, M. Rosa, J. Ugander, S. Vigna, Four degrees of separation 2012 [cited 2013 June 3]. Available from: http://avxiv.org/pdf/1111.4570.pdf

[21] P.V. Marsden, Homogeneity in confiding relations, Social Networks 10 (1988) $57-76$.

[22] I.S. Pool, M. Knochen, Contacts and influence, Social Networks 1 (1978) $5-51$. 
[23] E.M. Rogers, Diffusion of Innovation, The Free Press, 1983.

[24] D.H. Zanette, Dynamics of rumour propagation on small world networks, Physical Review E 65 (2002) 041908.

[25] Y. Moreno, M. Nekovee, A.F. Pacheco, Dynamics of rumour spreading in complex networks, Physical Review E 69 (2004) 066130.

[26] L.M.A Bettencourt, A. Cintron-Aries, D.I. Kaiser, The power of the spread of ideas from epidemiological models, Physica A 364 (2006) 513536.

[27] W.K. Ang, P.W. Jowitt, Path entropy method for multiple source water distribution networks, Engineering Optimization 37 (2005) 705-715.

[28] A. Yassin-Kassab, A.B. Temleman, T.T. Tankyimboh, Calculating maximum entropy flows in multi-source, multi-demand networks, Engineering Optimization 31 (1999) 695-729.

[29] M. Ruth, Information, order and knowledge in economic and ecological systems: implications for material and energy use, Ecological Economics 13 (1995) 99-114.

[30] R.E. Ulanowicz, Information theory in ecology, Computers and Chemistry 25 (2001) 393-399.

[31] C. Balocco, G. Grazzini, Sustainability and information in urban system analysis, Energy Policy 34 (2006) 2905-2914.

[32] Solar water heating program [Internet], Eskom Holdings SOC Limited [cited 2012 June 28], Available from: www.eskom.co.za/c/56/eskomsolar-water-heating-programme.

[33] HomeFlex Eskom Tariff [Internet], HomeFlex time of use tariff [cited 2013 June 3], Available from: www.eskom.co.za/content/Eskom\% 20Booklet.pdf 